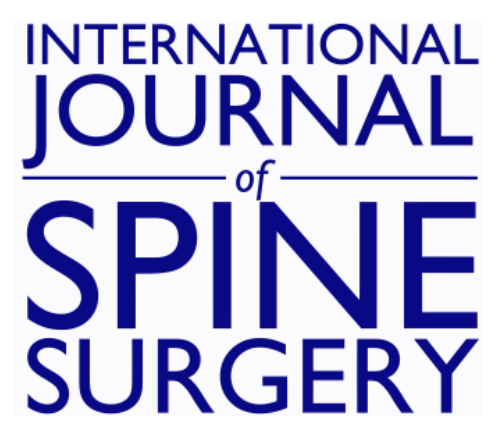

\title{
Surgeon Preference for Radiologist Interpretation of Deformity Radiographs--A Survey of Lumbar Spine Research Society Membership
}

CHRISTOPHER T. MARTIN, DAVID W. POLLY, JR and TAKASHI TAKAHASHI

Int J Spine Surg 2020, 14 (4) 527-533

doi: https://doi.org/10.14444/7069

http://ijssurgery.com/content/14/4/527

This information is current as of April 26, 2023.

Email Alerts Receive free email-alerts when new articles cite this article. Sign up at: http://ijssurgery.com/alerts 


\title{
Surgeon Preference for Radiologist Interpretation of Deformity Radiographs-A Survey of Lumbar Spine Research Society Membership
}

\author{
CHRISTOPHER T. MARTIN, MD, ${ }^{1}$ DAVID W. POLLY, JR, MD, ${ }^{1}$ TAKASHI TAKAHASHI, MD ${ }^{2}$ \\ ${ }^{I}$ The Department of Orthopaedic Surgery, University of Minnesota, Minneapolis, Minnesota, ${ }^{2}$ The Department of Radiology, University of Minnesota, \\ Minneapolis, Minnesota
}

\begin{abstract}
Background: The radiologist interpretation of scoliosis films is non-standardized, with some practitioners providing detailed measurements of the deformity whereas others defer the interpretation of the deformity to the ordering surgeon. For radiologists, the standard of care is not clear, and this creates confusion in terms of how much interpretation is required. However, detailed radiologist reports sometimes conflict with the surgeon's interpretation, which can create confusion for patients who receive the reports, or in extreme cases can lead to insurance denials. Thus, the purpose of this study was to help establish a standard for interpretation of these films by surveying the ordering surgeons and documenting expert opinion about the amount and type of radiologist interpretation that is requested.

Methods: We designed a SurveyMonkey survey which aimed to look at standard practice for radiologist dictation of scoliosis radiographs. Twelve questions were sent to Lumbar Spine Research Society membership via email with a description of the study. One follow-up email was also sent to non-responders.

Results: The rate of completed surveys was 46 out of 185 Lumbar Spine Research Society members (25\%). Thirty-seven respondents $(80 \%)$ worked in academic institutions, 33 were orthopedists $(71 \%)$, and 13 were neurosurgeons $(28 \%)$. Fifty percent reported that radiologists' level of detail in dictations was inconsistent at their institution. Detailed numeric reporting was rare $(6.5 \%)$. When the radiologist did provide numeric measurements, surgeons reported that they often differed from the surgeon's own measurements, with only $4 \%$ reporting that the measurements "rarely" differed from the surgeon's; 49\% reported that the radiologist's measurements that differed from the surgeon's had led to insurance denials for their patients. The majority of respondents $(70 \%)$ did not want the radiologist to provide detailed numeric measurements of the deformity, and $91 \%$ reported that the radiologist's measurements had no impact on their clinical decision making.

Conclusions: Detailed deformity measurements are time consuming for the radiologist, and would seem to have low clinical utility for the responding surgeons in this survey, with significant potential for discrepancies in interpretation to lead to insurance denials.
\end{abstract}

Level of Evidence: IV.

\section{Lumbar Spine}

Keywords: radiology, spinal deformity, adult spinal deformity, interpretation, scoliosis films, scoliosis radiographs, insurance denials, radiologist, surgeon preference

\section{INTRODUCTION}

Recent years have seen an increasing recognition of adult spinal deformity (ASD) as a cause of significant health-related disability. ${ }^{1-3}$ The current prevalence of scoliosis in the general population has been reported to be up to $32 \%,{ }^{2,4-6}$ with estimates being even higher (up to $68 \%$ ) in patients over $65 .^{7}$ By 2050 the US population aged 65 and older is projected to have doubled beyond current levels, ${ }^{8}$ and the prevalence of those with spinal deformity could exceed more than 60 million. $^{2}$ Thus, both radiologists and surgeons are likely to see ASD patients on an increasing basis in the near future.

Appropriate radiographic assessment of ASD patients requires full-length standing scoliosis radiographs. These images contain substantial skeletal detail given that they include a radiographic image of the entire axial skeleton. However, the amount of interpretation in the radiologist's dictation varies amongst providers. Detailed numeric measurements of the deformity are time consuming and risk 
conflicting with the surgeon-derived measurements, possibly leading to insurance denials. However, minimalist dictation risks providing an inappropriately sparse interpretation that may have no clinical utility. Thus, the amount of interpretation expected or required for these films is not clear.

In this study, we hoped to better define current surgeon preferences for interpretation of adult spinal deformity films through a survey of the membership of the Lumbar Spine Research Society (LSRS). The results should help to better inform appropriate clinical practice in this area.

\section{MATERIALS AND METHODS}

We designed a SurveyMonkey survey which aimed to look at standard practice for radiologist dictation of scoliosis radiographs (Supplemental Appendix, available online). A description of the study and a link to the survey was sent via email to members of the LSRS. The LSRS is an academic society consisting of lumbar spine surgeons who regularly treat patients with lumbar pathology, including patients with spinal deformity, and has 185 members. The survey consisted of 12 questions. One follow-up email was also sent to non-responders, which is the standard procedure for LSRSbased research surveys per the society's guidelines.

\section{RESULTS}

The initial survey was sent on July 2, 2018 and the email was opened by $58.2 \%$ of LSRS members, with $18.5 \%$ then opening the survey link. A follow-up email was then sent on July 17, 2018, which was opened by $45.6 \%$ of members, $10.7 \%$ of whom then opened the survey link. The survey was left open for 6 weeks to allow adequate time for responses. All responses were received within the first 3 weeks of the initial survey being sent. In total, 46 out of 185 LSRS members $(25 \%)$, completed all 12 questions from the survey.

\section{Demographics of Respondents}

Thirty-seven respondents $(80 \%)$ worked in academic institutions, with the remainder working in community or private practice settings. Thirty-three were orthopedists $(71 \%)$, and 13 were neurosurgeons $(28 \%)$. Twenty-six respondents $(56 \%)$ saw only adult spinal deformity patients, whereas 18 (39\%) saw both adult and pediatric patients, and 2 respondents $(4 \%)$ did not regularly see deformity patients. Eighteen of the respondents $(39 \%)$ had been in practice for more than 20 years, $9(20 \%)$ had been in practice 11 to 20 years, $8(17 \%)$ had been in practice 6 to 10 years, and 11 had been in practice 0 to 5 years $(24 \%)$.

\section{Radiologist Interpretation of Radiographs}

The majority of respondents reported that the amount of detail provided by the interpreting radiologist at their institution was inconsistent $(50 \%, 23$ respondents), or that the radiologist provided a generic description of the deformity without reporting detailed numeric measurements (39\%, 18 respondents). Detailed numeric reporting on the magnitude of the deformity in both the sagittal and coronal planes was rarely observed (6.5\%, 3 respondents).

When detailed numeric measurements were indeed provided by the radiologist, $43 \%$ of the respondents felt that the radiologist's interpretation frequently differed (more than $20 \%$ of the time) from the surgeon's interpretation whereas $20 \%$ felt that they often differed (10\%-20\% of the time), and only $4 \%$ felt that they rarely differed (less than $10 \%$ of the time). Fifteen respondents (33\%) were unable to answer this question because their radiologist never provided detailed numeric measurements (Figure 1 and Table 1).

In total, $39 \%$ of the respondents reported that differences between the radiologist's interpretation and the surgeon's interpretation of deformity radiographs had led to insurance denials for their patients (Figure 2 and Table 2). Three respondents $(6.5 \%)$ reported that this occurs commonly (more than $10 \%$ of cases), $9(19.5 \%)$ reported that it happens occasionally (5\%-10\% of cases), and 6 $(13 \%)$ reported that it happens rarely (less than $5 \%$ of cases). Forty-one percent reported that they had not seen insurance denials due to differences in interpretation. Notably, $9(19.5 \%)$ respondents were not able to comment on this question because their radiologists did not provide numeric measurements in any case. Thus, of the surgeons that did respond, 18 of $37(49 \%)$ had experienced insurance denials in at least some of their cases due to differences in interpretation between the radiologist's and surgeon's reporting on the deformity film.

\section{Surgeon Preferences}

Surgeons were asked how much interpretation of the deformity they wanted from their reporting 


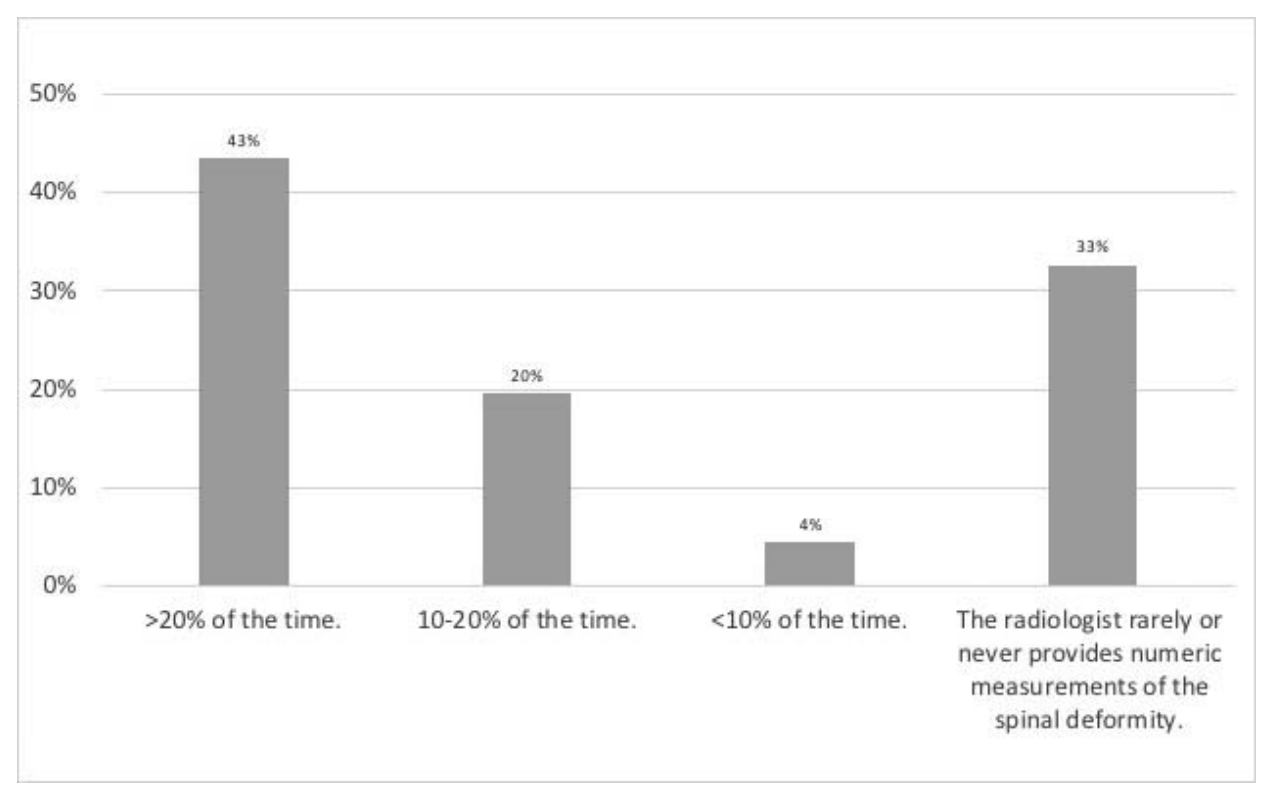

Figure 1. How often does the radiologist's deformity measurement substantially differ from the measurement done by the surgeon?

radiologist (Figure 3 and Table 3). A substantial majority $(70 \%)$ reported that they did not want detailed numeric measurements, with $50 \%$ wanting a generic description of the deformity without numeric interpretation, and $20 \%$ wanting no comment on the deformity at all. The remaining $30 \%$ of respondents requested detailed numeric reporting in both the sagittal and coronal planes.

The vast majority of the respondents (91\%), reported that the radiologist's interpretation had no impact on their clinical decision making (Figure 4 and Table 4). Four respondents (9\%) reported that while they did make their own numeric measurements, the radiologist's interpretation also played a role in their decision making. None $(0 \%)$ of the respondents relied solely on the radiologist interpretation for their clinical decision making.

Surgeons were then asked if they preferred a different type or amount of interpretation between pediatric and adult cases. The vast majority of respondents $(91 \%)$ requested that their adult and pediatric deformity films be treated in the same way by the interpreting radiologist. The specific type of

Table 1. How often does the radiologist's deformity measurement substantially differ from the measurement done by the surgeon?

\begin{tabular}{lr}
\hline Parameter & $\mathbf{\%}$ \\
\hline$>20 \%$ of the time & 43 \\
$10 \%-20 \%$ of the time & 20 \\
$<10 \%$ of the time & 4 \\
The radiologist rarely or never provides numeric measurements & 33 \\
of the spinal deformity. & 20 \\
Incidental findings only & \\
\hline
\end{tabular}

interpretation requested mirrored the response to the previous questions, with $31 \%$ requesting detailed numeric reporting for both adult and pediatric cases, and $61 \%$ requesting no detailed numeric reporting for either their adult or pediatric cases. Only 4 respondents $(9 \%)$ requested a different type or amount of numeric interpretation between adult and pediatric cases.

Surgeons were then asked to specify which measurements should be included when the radiologist did indeed provide detailed numeric measurements on adult spinal deformity films: $78 \%$ requested $\mathrm{C} 7$ sagittal vertical axis, $63 \%$ requested pelvic incidence-lumbar lordosis mismatch, 58\% requested lumbar lordosis, $58 \%$ requested the cobb angle of the coronal plane deformity, $45 \%$ requested pelvic tilt, $42 \%$ requested the global coronal balance, 39\% requested thoracic kyphosis, 33\% requested sacral slope, $21 \%$ requested the percentage of lumbar lordosis that exists between L4 and S1, 18\% requested T1-Pelvic Angle or global tilt, and 9\% requested the Scoliosis Research SocietySchwab classification.

\section{DISCUSSION}

Recent years have seen a substantial proliferation in the utilization of full-length standing scoliosis radiographs for assessment of ASD. Nonetheless, there is no consensus on the type or amount of radiologist interpretation that is appropriate for these types of films, leading to significant variability 


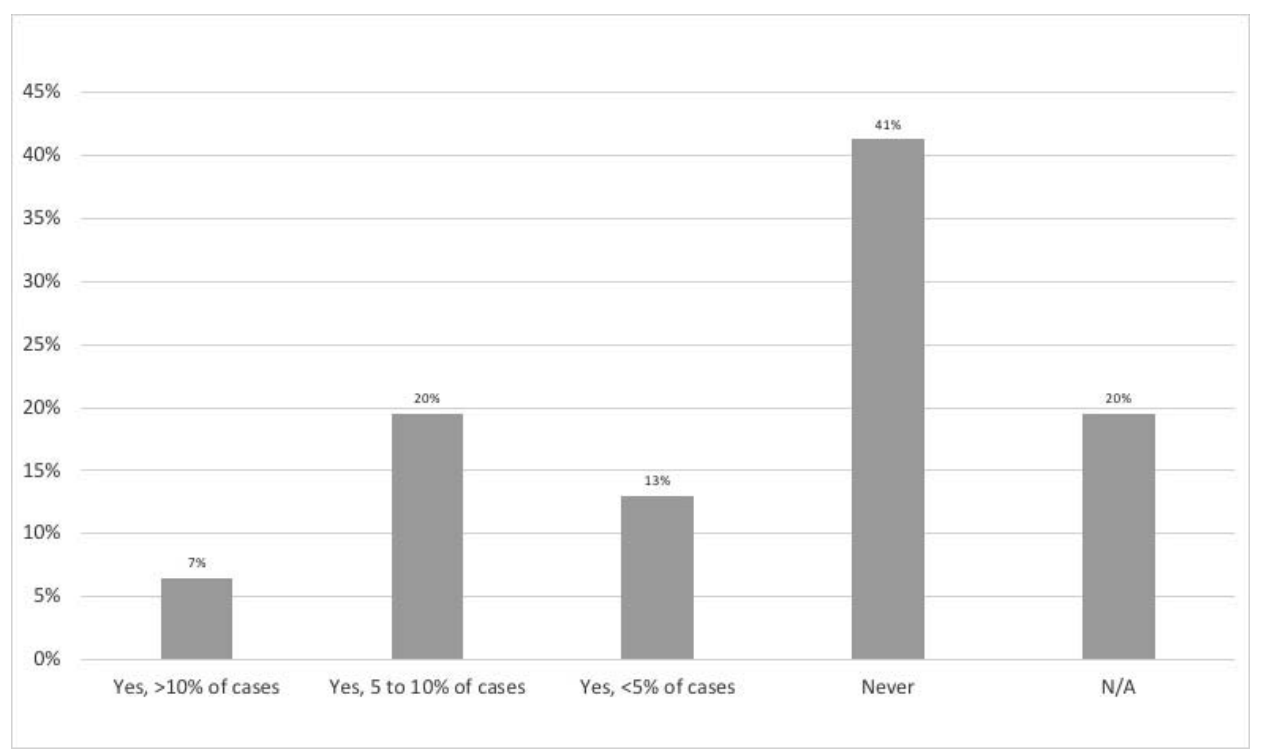

Figure 2. Sometimes the radiographic measurements taken by the surgeon differ from those reported by the radiologist. Has an insurance company denied one of your patients a surgery based on a radiologist interpretation of the deformity film?

between institutions and providers. Therefore, in this study, we hoped to help establish surgeon preferences for these films through a survey of the LSRS membership. Several of our findings merit further discussion.

Detailed numeric reporting risks conflicting with the surgeon's own interpretation of the deformity. In this survey, only $4 \%$ of respondents felt that the radiologist's interpretation "rarely" (less than 10\% of the time) differed from their own interpretation. Thus, differences in interpretation are common. At a minimum these discrepancies create confusion in the medical record, both for patients who have access to their records, as well as for the treating providers and for insurance companies who review the records. Insurance companies vary in their policies, but some favor the radiologist's interpretation, which raises the possibility of insurance denials due to discrepancies between the radiolo-

Table 2. Sometimes the radiographic measurements taken by the surgeon differ from those reported by the radiologist. Has an insurance company denied one of your patients a surgery based on a radiologist's interpretation of the deformity film?

\begin{tabular}{ll}
\hline Parameter & $\mathbf{\%}$ \\
\hline $\begin{array}{l}\text { Yes, differences in interpretation are a common occurrence } \\
\text { leading to insurance denials ( }>10 \% \text { of my cases). }\end{array}$ & 7 \\
$\begin{array}{l}\text { Yes, differences in interpretation occasionally lead to } \\
\text { insurance denials }(5 \%-10 \% \text { of my cases). }\end{array}$ & 20 \\
$\begin{array}{l}\text { Yes, the reads differ occasionally, but it has rarely led to } \\
\quad \text { issues with insurance approval ( }<5 \% \text { of my cases). }\end{array}$ & 13 \\
$\begin{array}{l}\text { No, although the reads sometimes differ I have never seen it } \\
\text { lead to an insurance denial. }\end{array}$ & 41 \\
My radiologist does not measure the deformity. & 20 \\
\hline
\end{tabular}

gist's and surgeon's opinions. Indeed, in cases where radiologists provide numeric measurements, $47 \%$ of surgeon respondents in this survey noted that the radiologist's interpretation had led to insurance denials at least some of the time. These insurance denials are a major headache for providers and for patients, result in delays in care, and require a significant time investment from the surgeon and their team to dispute. Thus, it is in the interest of all parties to minimize differences in interpretation of these adult deformity films.

If the radiologist's interpretation of ASD films had a high clinical utility, it might be worth working through these discrepancies. However, the vast majority of surgeons $(91 \%)$ reported that the radiologist's interpretation had no impact on their clinical decision making. This result is consistent with other studies which concluded that the radiologist's interpretation of fracture patterns in trauma ${ }^{9}$ and curve magnitude in adolescent scoliosis ${ }^{10}$ had low clinical utility. This question received the highest consensus on the survey, and thus the clinical utility of the radiologist's deformity interpretation seems to be low for most clinicians.

It is interesting to note that the vast majority of surgeon respondents $(91 \%)$ wanted the same type of radiologist interpretation on both adult and pediatric deformity films. The radiographic criteria that guide surgical decision making are different between adult and pediatric cases. In pediatric cases, the primary determinant is the magnitude and location 


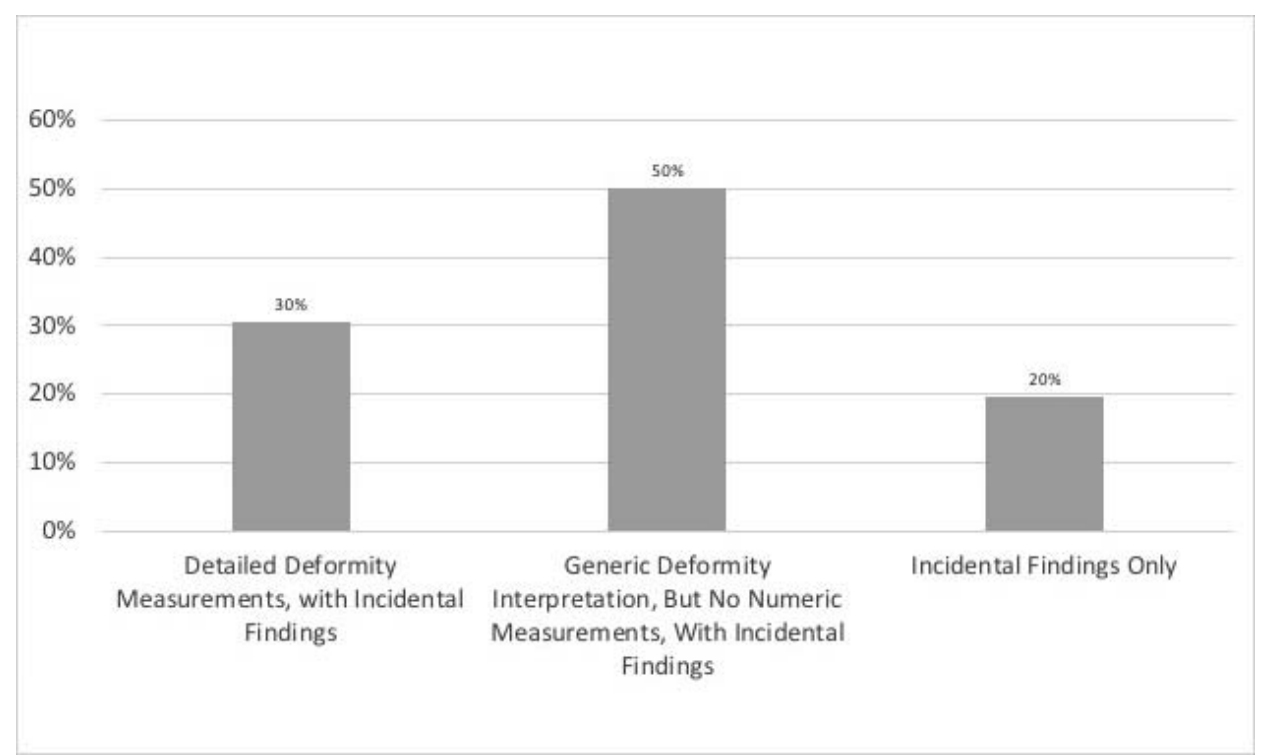

Figure 3. What information would be most useful to you, as the surgeon, in the radiologist's report?

of the coronal plane deformity, which is well illustrated in the widely accepted Lenke classification for adolescent idiopathic scoliosis. ${ }^{11}$ However, in adult cases there is an increased emphasis on the sagittal plane, as illustrated by the Scoliosis Research Society-Schwab classification. ${ }^{12}$ Thus, requesting uniform radiologist interpretation of both pediatric and adult patients does not truly reflect the nuanced differences between pediatric and adult surgical decision making. As noted previously, the same $91 \%$ of respondents felt that the radiologist's interpretation had no impact on their clinical decision making. It seems plausible that the request for a uniform interpretation of the films reflects this ambivalence towards the radiologist's interpretation.

Given surgeon's ambivalence towards the radiologist's interpretation, it is perhaps not surprising that surgeon preferences for the type and amount of interpretation was variable. Assuming that the radiologist planned to provide numeric interpretation of the deformity, surgeons were asked to list which measurements they would want included. Sagittal plane measurements were the most commonly requested, but there was no clear consensus,

Table 3. What information would be most useful to you, as the surgeon, in the radiologist's report?

\begin{tabular}{lc}
\hline Parameter & $\mathbf{\%}$ \\
\hline Detailed deformity measurements, with incidental findings & 30 \\
Generic deformity interpretation, but no numeric measurements, & 50 \\
$\quad$ with incidental findings & 20 \\
Incidental findings only & 20 \\
\hline
\end{tabular}

and a significant number of surgeons (22\%), requested every possible measurement listed. Requesting detailed numeric reporting on a large number of numeric measurements does not seem reasonable, given the apparent low clinical utility and possibility for conflict with the surgeon's measurements. Furthermore, this type of detailed reporting on a large number of measurements is time consuming for the radiologist. Most radiologists are compensated per image read, and thus there is a strong financial disincentive to perform large numbers of detailed measurements, particularly when the results have low clinical utility for the ordering surgeon. We would suggest that surgeons meet with the radiologists at their institution and create an agreed upon template for deformity image dictations. These templates can help insure that the ordering providers receive the appropriate amount and type of requested interpretation, and decrease confusion for the radiologists.

This study does have several limitations. Firstly, the number of respondents (46) is small, and represents only $25 \%$ of the total LSRS membership. A response rate of $25 \%$ is typical of email-based survey studies. Further, the LSRS is an academic society, and its membership is representative of the thought leaders in lumbar spine study. Nonetheless, it is possible that the opinions of this small group do not reflect wider clinical practice, and these small numbers limit the generalizability of this study. Secondly, this survey was designed to assess surgeons' opinions of the radiologists' interpreta- 


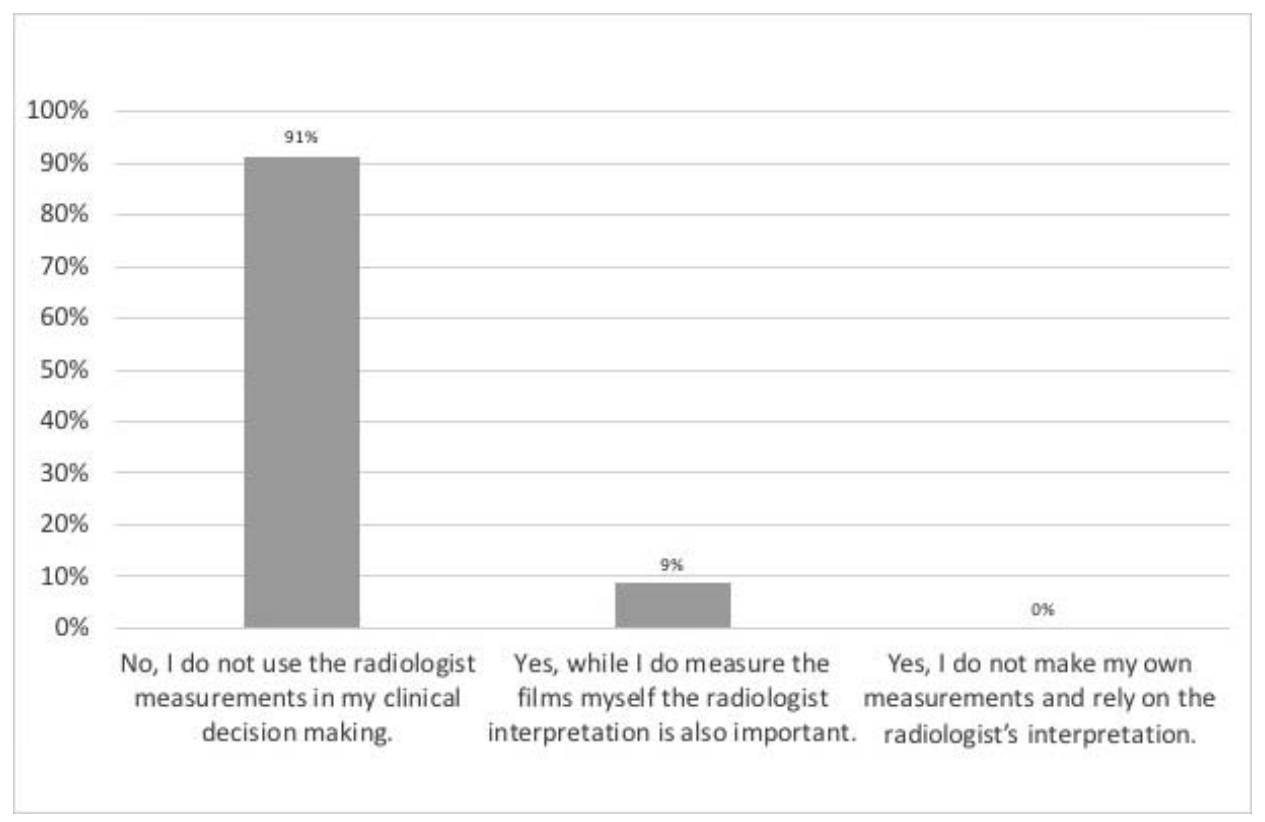

Figure 4. As the treating surgeon, does the radiologist's interpretation of the spinal deformity impact your decision about what treatment to offer the patient?

tions specifically on the spinal deformity. It was not designed to determine whether or not a radiologist should provide other forms of interpretation on these radiographs. Radiologists comment on more than just the deformity when they review a spine radiograph. ${ }^{10}$ They are responsible for listing incidental extraspinal findings, which are often missed by the ordering surgeons; for commenting on the technical quality of the films; for training the technologists who obtain the films; and for maintaining safety standards. ${ }^{10}$ Each of these items has value separate from the interpretation of the deformity, and where appropriate could be included on a reasonable dictation template. Thus, our study is not a commentary on which type of provider (surgeon or radiologist) should interpret these films overall, but rather specifically looks at surgeon preferences on interpretation of the spinal deformity.

In summary, detailed numeric reporting on the magnitude of the spinal deformity by the interpreting radiologist poses several challenges. These

Table 4. As the treating surgeon, does the radiologist's interpretation of the spinal deformity impact your decision about what treatment to offer the patient?

\begin{tabular}{lr}
\hline Parameter & $\mathbf{\%}$ \\
\hline $\begin{array}{l}\text { No, I do not use the radiologist's measurements in my } \\
\text { clinical decision making. }\end{array}$ & 91 \\
$\begin{array}{l}\text { Yes, while I do measure the films myself the radiologist's } \\
\quad \text { interpretation is also important. }\end{array}$ & 9 \\
$\begin{array}{l}\text { Yes, I do not make my own measurements and rely on the } \\
\text { radiologist's interpretation. }\end{array}$ & 0 \\
\hline
\end{tabular}

measurements are time consuming and financially disincentivized, frequently differ from the surgeon's own measurements, can lead to insurance denials, and rarely have an impact on clinical decision making. Numerous measurements exist, and there is no consensus amongst surgeons on which types surgeons would want to be included by the radiologist. Given these findings, we would suggest that surgeons work with the radiologists at their institution to develop a standard template for interpretation of ASD films at their institution that either does not include or minimizes the reporting of detailed numeric deformity measurements. A standardized template would help decrease variability in interpretation across providers, and would help ensure that the ordering clinician receives the information that they actually want or need for appropriate patient care.

\section{REFERENCES}

1. Bess S, Line B, Fu KM, et al. The health impact of symptomatic adult spinal deformity: comparison of deformity types to United States population norms and chronic diseases. Spine (Phila Pa 1976). 2016;41(3):224-233.

2. Ames CP, Scheer JK, Lafage V, et al. Adult spinal deformity: epidemiology, health impact, evaluation, and management. Spine Deform. 2016;4(4):310-322.

3. Good CR, Auerbach JD, O'Leary PT, Schuler TC. Adult spine deformity. Curr Rev Musculoskelet Med. 2011;4(4):159167.

4. Hong JY, Suh SW, Modi HN, Hur CY, Song HR, Park JH. The prevalence and radiological findings in 1347 elderly 
patients with scoliosis. J Bone Joint Surg Br. 2010;92(7):980983.

5. Francis RS. Scoliosis screening of 3,000 college-aged women. The Utah Study — phase 2. Phys Ther. 1988;68(10):15131516.

6. Carter OD, Haynes SG. Prevalence rates for scoliosis in US adults: results from the first National Health and Nutrition Examination Survey. Int J Epidemiol. 1987;16(4):537-544.

7. Schwab F, Dubey A, Gamez L, et al. Adult scoliosis: prevalence, SF-36, and nutritional parameters in an elderly volunteer population. Spine (Phila Pa 1976). 2005;30(9):10821085 .

8. Vincent GK Velkoff VA. The Next Four Decades, the Older Population in the United States: 2010 to 2050. Current Population Reports. Washington, DC: United States Census Bureau. Publication 25-1138.

9. Clark R, Anderson MB, Johnson BH, Moore DE, Herbert FD. Clinical value of radiologists' interpretations of perioperative radiographs of orthopedic patients. Orthopedics. 1996;19(12):1003-1007.

10. Crockett HC, Wright JM, Burke S, Boachie-Adjei O. Idiopathic scoliosis. The clinical value of radiologists' interpretation of pre- and postoperative radiographs with interobserver and interdisciplinary variability. Spine (Phila Pa 1976). 1999;24(19):2007-2009; discussion 2010.

11. Lenke LG, Betz RR, Harms J, et al. Adolescent idiopathic scoliosis: a new classification to determine extent of spinal arthrodesis. J Bone Joint Surg Am. 2001;83-A(8):11691181.

12. Schwab F, Ungar B, Blondel B, et al. Scoliosis Research
Society-Schwab adult spinal deformity classification: a validation study. Spine (Phila Pa 1976). 2012;37(12):1077-1082.

Disclosures and COI: None of the authors have relevant disclosures related to this manuscript. Each author certifies that he or she has no commercial associations (eg, consultancies, stock ownership, equity interest, patent/licensing arrangements, etc) that might pose a conflict of interest in connection with the submitted article. This paper is exempt from Institutional Review Board review as it is not human-subjects research. There was no external source of funding for this study.

Corresponding Author: Christopher T. Martin, MD, Department of Orthopaedic Surgery, University of Minnesota, 2512 South 7th Street R200-Location, Minneapolis, MN 55454. Phone: (612) 273-7951 Email: mart1865@umn.edu.

Published 28 August 2020

This manuscript is generously published free of charge by ISASS, the International Society for the Advancement of Spine Surgery. Copyright (c) 2020 ISASS. To see more or order reprints or permissions, see http://ijssurgery.com. 